\title{
The effect of Trypanosoma evansi infection on pig performance and vaccination against classical swine fever
}

\author{
W.G. Holland ${ }^{\mathrm{a}, \mathrm{b}}$, T.T. Do ${ }^{\mathrm{b}}$, N.T. Huong ${ }^{\mathrm{b}}$, N.T. Dung ${ }^{\mathrm{b}}$, \\ N.G. Thanh ${ }^{\mathrm{b}}$, J. Vercruysse ${ }^{\mathrm{a}}$, B.M. Goddeeris ${ }^{\mathrm{a}, \mathrm{c}, *}$ \\ ${ }^{a}$ Ghent University, Faculty of Veterinary Medicine, Department Virology-Parasitology-Immunology, \\ Salisburylaan 133, B-9820 Merelbeke, Belgium \\ ${ }^{\mathrm{b}}$ National Institute of Veterinary Research, Truong Chinh Street 86, Dong Da, Hanoi, Viet Nam \\ ${ }^{\mathrm{c}}$ Faculty of Agriculture and Applied Biological Sciences, Department of Animal Sciences, K.U. Leuven, \\ Kasteelpark Arenberg 30, 3001 Leuven, Belgium
}

Received 14 June 2002; accepted 22 October 2002

\begin{abstract}
Although Trypanosoma evansi is not considered as an important pathogen in pigs, it may interfere with other pathogens or vaccinations by its immunosuppressive nature. In order to determine whether T. evansi alters pig performance and induces immunosuppression in pigs, induction of immune responses by vaccination against classical swine fever (CSF) and by immunization with a control antigen, human serum albumin (HSA), was assessed in T. evansi-infected and non-infected animals. Although $T$. evansi infection did not have a significant influence on growth performance, feed conversion or PCV, antibody responses against both the test antigen HSA and the CSF vaccine were significantly reduced in $T$. evansi-infected animals as compared to uninfected animals. Moreover, the reduced response against the CSF vaccine appears to be accompanied by a less well-developed protection against CSF with higher fever responses and leukopenia. This immunosuppression might explain the accounts of poor protection of CSF-vaccinated pigs reported in T. evansi-endemic areas of Vietnam, and suggests that prior treatments with trypanocidal drugs to improve the efficacy of CSF vaccination, may be justified.

(C) 2002 Elsevier Science B.V. All rights reserved.
\end{abstract}

Keywords: Immunosuppression; Classical swine fever; Trypanosoma evansi; Pig-protozoa

\footnotetext{
* Corresponding author. Tel.: +32-16-321-427; fax: +32-16-321-994.

E-mail address: bruno.goddeeris@agr.kuleuven.ac.be (B.M. Goddeeris).
} 


\section{Introduction}

Infections with Trypanosoma evansi in pigs are not conceived important and rarely reports of diseased pig herds are received. So far a limited number of studies has been carried out in South-east Asia on T. evansi in pigs with various outcomes. Arunasalam et al. (1995) described an outbreak in breeding stock characterized by fever, anorexia, emaciation, abortion and death. Bajyana Songa et al. (1987) noticed also reproductive problems in Thailand while Reid et al. (1999) concluded that the major implication of T. evansi infection in pigs laid in their hosting as a reservoir for other livestock species as cattle, horses and buffaloes.

Similar as the situation in water-buffaloes, where vaccination failures were described against haemorrhagic septicaemia with a Pasteurella vaccine (Holland et al., 2001b), recently a large number of cases of insufficient protection induced by different commercial classical swine fever (CSF) vaccines were reported in Vietnam; vaccinated pigs died with clear symptoms of acute CSF despite being vaccinated. CSF is considered to be one of the most important infectious diseases affecting pigs in many countries in Asia and elsewhere, with numerous outbreaks all year round (Dung and Blacksell, 2000).

Many possibilities for vaccination failures do exist such as vaccine quality, vaccination technique and quality of cold chain. Although vaccination failures due to trypanosome infections have been described in ruminants, so far no investigations were carried out on the possible immunosuppressive role of trypanosome infections in pigs (Whitelaw et al., 1979; Ilemobade et al., 1982; Rurangirwa et al., 1983; Ikeme et al., 1984; Onah et al., 1997, 1998; Holland et al., 2001b).

The main objective of the present study was to investigate if T. evansi induces immunosuppression in pigs. To determine whether T. evansi induces immunosuppression and may interfere with the development of immunity after vaccination in pigs, $T$. evansi-infected and non-infected pigs were vaccinated against CSF and subsequently challenged with virulent CSF virus. Fever, survival rate, leukocyte and trombocyte counts and specific CSF antibody titres were monitored. All animals received also two immunizations with human serum albumin (HSA) as a control antigen and antibody titres against HSA were monitored. Additionally, we examined the pathological importance of T. evansi in pigs. So far several studies were undertaken to investigate the influence of $T$. evansi infection on production in livestock (Payne et al., 1991, 1992, 1993; Pohlpark et al., 1999). However, no such studies were conducted in pigs and to further elaborate on the possible pathological role of T. evansi in pigs, daily weight gain, feed conversion and packed cell volume (PCV) of infected and non-infected pigs were monitored after an experimental infection.

\section{Materials and methods}

\subsection{Experimental animals and design}

A total of 21 piglets of mixed sex, 5 weeks of age, and confirmed negative for T. evansi and CSF virus-specific antibodies (ELISA according to Verloo et al., 2000 and Colijn et al., 1997) were selected. After 3 days acclimatisation, animals were allotted into three equal groups $(n=7)$. The three groups were housed separately and animals were equally 
fed on a restricted diet of commercially available concentrate, the daily amount recorded. Groups A and $\mathrm{C}$ were infected with $10^{6}$ T. evansi bloodstream trypamastigotes of the isolate WH ITMAS 101298 (Holland et al., 2001a) while group B was the non-infected control group. Four weeks after infection with T. evansi, animals of group A and control group B were vaccinated IM in the right neck, with the commercial CSF vaccine Pestiffa ${ }^{\circledR} \mathrm{C}$ strain (Lot no. L73452, Merial, France). Further, all three groups received an IM immunisation in the left neck site with $1 \mathrm{mg}$ HSA (Sigma A-3782). Hence, $1 \mathrm{mg}$ of HSA was dissolved in $0.5 \mathrm{ml}$ saline and subsequently mixed with $0.5 \mathrm{ml}$ Freund's incomplete adjuvant. Four weeks after the first immunisation, all animals received a booster with HSA. Eight weeks after the CSF vaccination, or 12 weeks after $T$. evansi infection, all three groups were challenged intra-nasally with a virulent dose of a CSF strain isolated from Vietnam (Ky Son district, Nghe An province). Therefore, heparinised whole blood was collected from a viraemic experimentally infected pig.

\subsection{Sampling procedures}

Plain and heparinised blood samples of all pigs were collected weekly until the CSF virus challenge to confirm the presence or absence of parasites and to determine the PCV and antibodies levels against CSF virus and HSA. T. evansi-infected animals were monitored daily for fever, anorexia and survival rate. All animals were weighed every fortnight.

After challenge with the CSF virus, blood samples were collected in EDTA at days 0, 2, 4, 7, 10 and 14 post-challenge to determine the number of leukocytes and trombocytes. After challenge, animals were examined daily for body temperature (fever $>40^{\circ} \mathrm{C}$ ) and clinical symptoms of CSF, e.g. anorexia, paresis.

\subsection{Laboratory procedures}

\subsubsection{Detection of T. evansi infections}

To check animals for the presence of T. evansi, heparinised blood was analysed with the micro-haematocrite centrifugation technique (MHCT; Van Meirvenne, 1999) and meanwhile the PCV was recorded. Provided the MHCT was negative, heparinised blood $(0.25-$ $0.5 \mathrm{ml}$ ) was inoculated intra-peritoneally into laboratory bred white mice. The inoculated mice were bled from the tail and a wet blood film was prepared to detect parasitaemia. Mice were checked two-three times a week for 1 month. Antibodies against T. evansi were measured by ELISA on T. evansi VAT RoTat 1.2 (Verloo et al., 2000) with minor modifications; test sera were diluted 1/400 in PBS-blotto and rabbit anti-pig IgG peroxidase (Sigma A-5670) diluted 1/15000 in PBS-Tween 20 was used as a conjugate.

\subsubsection{CSF-specific antibodies}

Sera were tested with the Ceditest ${ }^{\circledR}$ CSF virus, a complex-trapping blocking ELISA that uses two monoclonal antibodies directed against the recombinant CSFV E2 antigen (Colijn et al., 1997). ELISA results were expressed as the percentage inhibition $(I(\%)=100 \times$ ( $1-$ [OD test serum - mean OD positive reference serum $] /[$ mean OD negative reference serum - mean OD positive reference serum]) and a cut-off value of $30 \%$ was applied: $I$ $(\%)>30 \%$ was considered positive, $I(\%)<30 \%$ was considered negative. All test sera were 
tested undiluted and diluted two-fold in PBS (from 1:2 to 1:8). The titre of a test serum was the inverse of the highest dilution with $I(\%)>30 \%$. In order to detect significant differences between the two groups, the mean titre of each group was calculated and analysed by ANOVA.

\subsubsection{HSA-indirect antibody ELISA}

The ELISA was previously described by Holland et al. (2001b) and slightly modified. In short, ELISA plates were coated overnight with HSA (Sigma A-3782) diluted in PBS to a concentration of $2 \mu \mathrm{g} / \mathrm{ml}$ and, the next day, blocked and subsequently washed. All test sera collected post-immunisation were diluted in PBS starting at 1/1000 and subsequent two-fold dilutions were prepared until 1/1,024,000. After washing, rabbit anti-pig IgG (whole molecule)-peroxidase (Sigma A-5670) diluted 1/10,000 in PBS was added. As a substrate chromogen ABTS solution was used. The titre of a test serum was the inverse of the dilution having an OD higher or equal to the calculated cut-off. The latter consisted of the mean OD of all pre-immunisation sera $(n=21)$ at dilution $1 / 1000$, plus three times the standard deviation (cut-off $=0.22 \mathrm{OD}$ ). In order to detect significant differences between the two groups, the mean titre of each group was calculated and analysed by ANOVA.

\subsubsection{Leukocyte and trombocyte counts}

The number of leukocytes and tromobocytes in EDTA blood samples was determined in a Sysmex ${ }^{\circledR}$ KX21 Coulter counter. Leukopenia was defined as $<8 \times 10^{6}$ cells $/ \mathrm{ml}$ and trombocyopenia as $<200 \times 10^{6}$ cells $/ \mathrm{ml}$.

\subsubsection{Fluorescence antibody test}

To confirm the presence of CSF virus antigen in tissue biopsies from slaughtered animals, the fluorescence antibody test (FAT) was used as described by OIE (1996).

\section{Results}

\subsection{T. evansi screening and infection}

All T. evansi-injected animals (groups A and C) were confirmed parasitologically positive from the first week post-infection onwards, while all animals of the control group remained negative throughout the experiment. From 9 weeks post-infection onwards some infected animals could not be confirmed parasitologically positive anymore. All pigs of groups $\mathrm{A}$ and $\mathrm{C}$ became serologically positive (T. evansi-specific antibodies) from day 7 post-infection onwards, while animals of group B remained negative. During the first 12 weeks post-infection, no clinical signs of $T$. evansi infection were observed.

\subsection{Growth, feed conversion and PCV}

No significant differences in daily weight gain and feed conversion between infected and non-infected animals were observed. The 14 pigs infected with $T$ evansi (groups A and C) 
gained an average of $19.6 \mathrm{~kg}$, while the non-infected pigs (group B) gained $20.4 \mathrm{~kg}$ over the same period until challenged with the CSF virus. The infected and non-infected groups had over the 12 weeks period feed conversions of 2.85 and 2.80 , respectively.

During the 12-week monitoring period no significant differences in packed cell volume between the groups could be observed: the mean PCV levels remained stable around 35, with no major fluctuations in either group.

\subsection{Clinical symptoms after CSF virus challenge}

One animal of the B group succumbed after chronic diarrhoea (Clostridia spp.) on the day of challenge. After the CSF virus challenge, three out of seven animals in the non-vaccinated T. evansi-infected group $\mathrm{C}$ were found recumbent and were subsequently euthanised on days 2,7 and 8, respectively. The FAT on spleen samples of these three pigs were positive for CSF. The other four animals in this group lost their appetite and were dull for a number of days. In the vaccinated T. evansi-infected group A, four animals had reduced appetite for 2-5 days after challenge. In the vaccinated uninfected group B, however, no symptoms were observed.

Body temperature was recorded daily for 14 days after CSF challenge. The percentage of recordings of animals with fever $\left(>40^{\circ} \mathrm{C}\right)$ over this 14 -day period was 22,11 and 42 for groups A, B and C, respectively. The daily percentages of animals with fever are represented in Fig. 1. Upon CSF challenge, more animals with fever were detected in the $T$. evansi-infected groups A and $\mathrm{C}$ than in the non-infected group B, and could be detected for a longer time. For the CSF-vaccinated groups, more animals with fever were detected in the T. evansi-infected group A than in the non-infected group B and also for a longer time (Fig. 1).

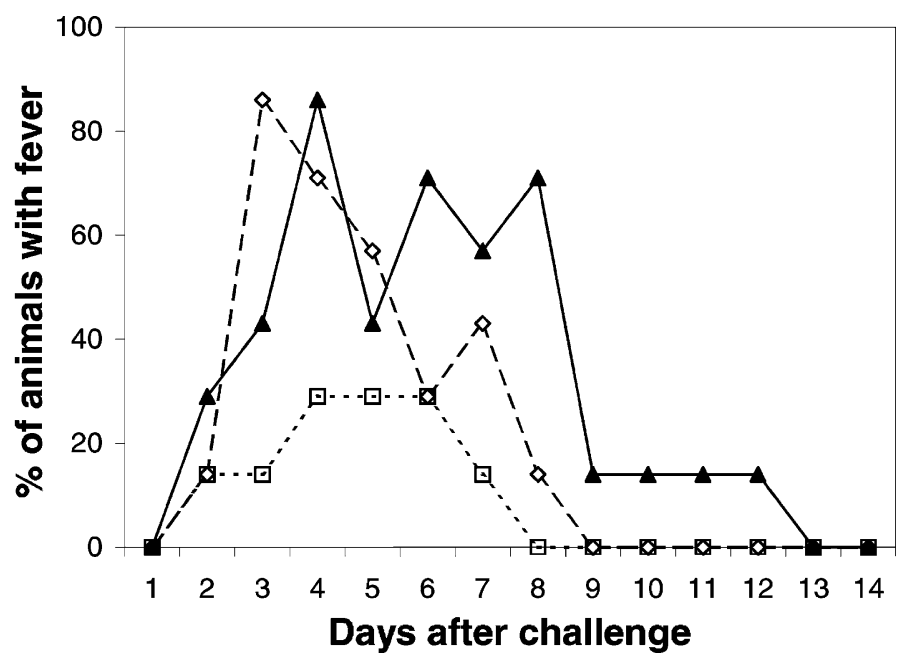

Fig. 1. Percentage (\%) of animals per day with fever after CSFV challenge. T. evansi-infected and CSF-vaccinated (group A $(\diamond)$ ), only CSF vaccinated (group B $(\square)$ ) and only T. evansi-infected animals (group C $(\boldsymbol{\Delta})$ )). 


\subsection{Leukocyte, thrombocyte counts}

In group A, no leukopenia could be detected throughout the 14 day monitoring period after the CSF virus challenge. One animal of group B had leukopenia on day 4 and recovered on day 7. In group C, three animals were found with leukopenia; one on day 4 and one on day 7 , both succumbing the same day, while the third animal was found with leukopenia on day 10 but fully recovered on day 14 .

Thrombocytopenia was found in group A in two animals on days 2 and 4, one animal only on day 2 and one animal only on day 4 . In group B, low thrombocyte levels were found, in one animal on days 2 and 4, one animal on days 4 and 7 and one animal only on day 2. In group $\mathrm{C}$, low thrombocytopenia levels were found in two animals on days 2 and 4 of which one died, one animal on days 2, 4 and 7 which subsequently succumbed and, finally one animal on days 4 and 7 which also died.

\subsection{CSF-specific antibodies}

All pigs vaccinated with the CSF vaccine (groups A and B) sero-converted as measured by the Ceditest ${ }^{\circledR}$ CSFV. CSF-specific antibody titres increased gradually from day 14 onwards to maximum levels at day 35 . On day 28,35 and 42 post-vaccination, titres were significantly higher in the non-infected group B than the infected group A (Fig. 2). Animals of the non-vaccinated control group remained sero-negative until the day of CSF-challenge.

\subsection{HSA-indirect antibody ELISA}

Upon HSA-immunization, all 21 pigs sero-converted against HSA as measured by ELISA. After primary immunisation the mean titres of the T. evansi-infected groups A and $\mathrm{C}$ and the uninfected group B increased gradually in both groups with a non-significantly higher

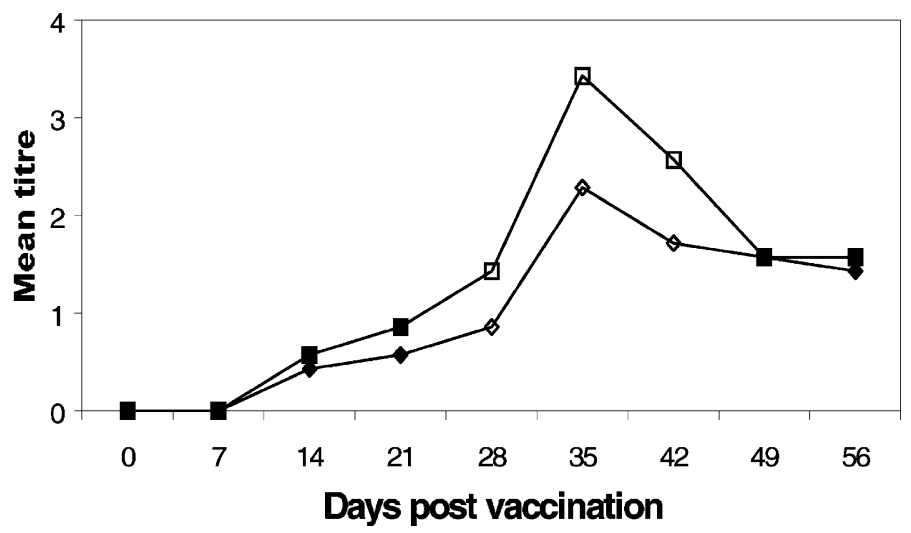

Fig. 2. CSF antibody titres determined by the Ceditest ${ }^{\circledR}$ CSFV of T. evansi-infected (group A $(\diamond)$ ) and uninfected pigs (group B $(\square)$ ) after vaccination with Pestiffa ${ }^{\circledR}$ C strain. Open symbols indicate statistically significant differences $(P<0.05)$. 


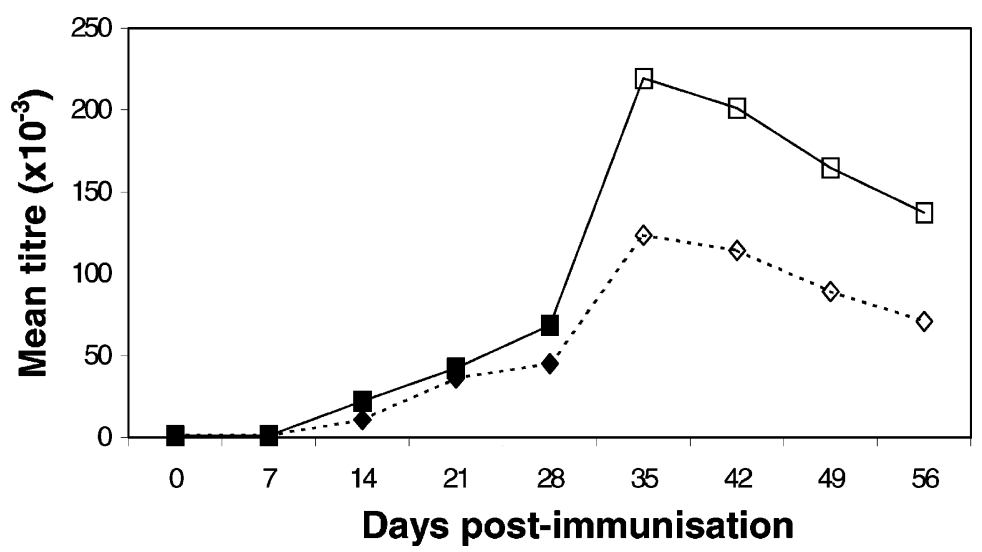

Fig. 3. HSA-specific antibody titres of T. evansi-infected (groups A and C $(\diamond)$ ) and uninfected pigs (group B ( $\square$ )) after primary (day 0) and secondary (day 28) HSA immunisation. Open symbols indicate statistically significant differences $(P<0.05)$.

level in the B group (Fig. 3). Following the booster immunisation at day 28, the mean titres for both infected and non-infected groups increased abruptly, with at all time points significantly higher levels for the uninfected group B than the infected groups A and C.

\section{Discussion}

The experimental infections of pigs with the T. evansi buffalo strain WH ITMAS 101298 did not induce major clinical symptoms: growth performance, feed conversion and PCV levels were not affected significantly compared to the uninfected controls. The absence of clinical symptoms or only low influence on growth, feeding or PCV levels confirms findings by others (Dr. Phan Dich Lan, Vietnam, personal communication, Reid et al., 1999) that T. evansi experimental infection in (young) pigs has a limited pathology. Other studies, however, investigating the influence of natural T. evansi infection on pig production, demonstrated fertility problems (Arunasalam et al., 1995; Bajyana Songa et al., 1987). It should, however, be noted that all experimental infections including the present study, were carried out on young animals with non-pig isolates.

The challenge with the CSF virus was lethal for three out of seven non-vaccinated pigs while the remaining four animals had fever for at least three days post-challenge. Also the percentage of recordings of thrombocytopenia in the non-vaccinated group was nearly twice (32\%) those of the vaccinated groups A and B (17\%). Conversely, leukopenia appeared not to be of major importance for the challenged non-vaccinated group. On the contrary, all the vaccinated animals survived the virus challenge confirming the protective value of the vaccine that prevents mortality and major clinical symptoms. However, some animals in the T. evansi-infected group A showed loss of appetite for 2-5 days post challenge.

The present study demonstrates that the presence of a $T$. evansi infection lowers the immunoresponsiveness of fattening pigs to concurrent immunisations. Indeed antibody 
responses against both the test antigen HSA and the CSF vaccine were significantly reduced in T. evansi-infected animals as compared to uninfected animals. This reduced response against the CSF vaccine appears to be accompanied with a less well-developed protection against CSF. A more profound loss of appetite post-challenge and twice the number of days with fever were observed in the T. evansi-infected group as compared to the non-infected group. However, no differences in leukopenia, trombocytopenia or survival rates were observed between the groups.

These data support the findings in ruminants on the immunosuppressive nature of trypanosome infections (Whitelaw et al., 1979; Ilemobade et al., 1982; Rurangirwa et al., 1983; Ikeme et al., 1984, Onah et al., 1998, Holland et al., 2001b). In mice and cattle (Sileghem et al., 1991; Sileghem and Flyn, 1992), it has been demonstrated that Trypanosoma infections reduce $\mathrm{T}$ cell responsiveness. In both the species, $\mathrm{T}$ cell proliferation was profoundly suppressed upon mitogenic stimulation and mediated by macrophage-like suppressor cells, leading to suppressed interleukin-2 secretion and impaired expression of the interleukin-2 receptor. A possible explanation for the reduced antibody responses in our infected pigs might thus reside in a $\mathrm{T}$ cell suppression induced by the concurrent $T$. evansi infection. Indeed, the induction of a good antibody response needs a good $\mathrm{T}$ helper cell response as the latter provides help to the B cell though delivery of cytokines.

In conclusion, our results suggest that the inductive capacity to mount immune responses on heterologous antigens may be suppressed in T. evansi-infected fattening pigs and consequently that $T$. evansi infection might interfere with the development of protective immunity upon heterologous vaccinations. This immunosuppression may explain the accounts of poor protection of CSF-vaccinated pigs reported in T. evansi-endemic areas of Vietnam. Therefore, it is suggested that in T. evansi-endemic areas a treatment with trypanocidal drugs may enhance the efficacy of CSF vaccination.

\section{Acknowledgements}

The study was carried out within the framework of the Vietnamese-Belgium project "Strengthening the National Institute of Veterinary Research in Central and North Vietnam" (University of Ghent-Flemish Interuniversity Council) at the National Institute of Veterinary Research, Hanoi, Vietnam. The Ceditest ${ }^{\circledR}$ CSFV was kindly donated by ID-Lelystad, The Netherlands.

\section{References}

Arunasalam, V., Chandrawathani, P., Sivanandan, S., 1995. An outbreak of Trypansoma evansi infection in pigs. J. Vet. Malaysia 7, 71-73.

Bajyana Songa, E., Hamers-Castermann, C., Hamers, S., Pholpark, M., Pholpark, S., Leidl, K., et al., 1987. The use of the card agglutination test (TESTRYP CATT) for the detection of Trypanosoma evansi infection a comparison with other trypanosomiasis diagnostic tests under field conditions. Ann. Soc. Belge Méd. Trop. 67, 137-148.

Colijn, E.O., Bloemraad, M., Wensvoort, G., 1997. An improved ELISA for the detection of serum antibodies directed against classical swine fever virus. Vet. Microbiol. 59, 15-25. 
Dung, N.T., Blacksell, T.D., 2000. Pig production and classical swine fever in Vietnam. In: Proceedings on Classical Swine Fever and Emerging Diseases in Southeast Asia. ACIAR Proc. 94, 126-127.

Holland, W.G., Claes, F., My, L.N., Thanh, N.G., Tam, P.T., Verloo, D., et al., 2001a. A comparative evaluation of parasitological tests and a PCR for Trypanosoma evansi diagnosis in experimentally infected water buffaloes. Vet. Parasitol. 97, 23-33.

Holland, W.G., My, L.N., Dung, T.V., Thanh, N.G., Tam, P.T., Vercruysse, J., et al., 2001b. The influence of Trypanosoma evansi infection on the immunoresponsiveness of experimentally infected water buffaloes. Vet. Parasitol. 102, 225-234.

Ikeme, M.M., Aziz Saharee, A., Zamri Saad, M., Koran, R., 1984. Effect of experimental Trypanosoma evansi infections in cattle and subsequent response to Haemorrhagic Septicaemia vaccine, Trop. Vet. 2, 61-67.

Ilemobade, A.A., Adegboye, D.S., Onovrian, O., Chima, J.C., 1982. Immunodepressive effects of trypanosomal infection in cattle immunised against contagious bovine pleuropneumoniae. Parasite Immunol. 4, $273-282$.

OIE, 1996. Classical swine fever (hog cholera). In: Manual of standards for diagnostic tests and vaccines. Office International des Epizooties, Paris, pp. 145-154.

Onah, D.N., Hopkins, J., Luckins, A.G., 1997. Effects of Trypanosoma evansi on the output of cells from a lymph node draining the site of Pasteurella haemolytica vaccine administration. J. Comp. Pathol. 117, 73-82.

Onah, D.N., Hopkins, J., Luckins, A.G., 1998. Increase in CD5+ B cells and depression of immune responses in sheep infected with Trypanosoma evansi. Vet. Immunol. Immunopathol. 63, 209-222.

Payne, R.C., Djauhari, D., Partoutomo, S., Jones, T.W., Pearson, R.A., 1991. Trypanosoma evansi infection in worked and unworked buffaloes (Bubalus bubalis) in Indonesia. Vet. Parasitol. 40, 197-206.

Payne, R.C., Sukanto, I., Partoutomo, S., Polytedi, F., 1992. Experimental infection of Friesian Holstein calves with an Indonesian isolate of Trypanosoma evansi, Trop. Med. Parasitol. 115-117.

Payne, R.C., Sukanto, I., Bazely, K., Jones, T.W., 1993. The effect of Trypanosoma evansi infection on the oestrus cycle of Friesian Holstein heifers. Vet. Parasitol. 51, 1-11.

Pohlpark, S., Pohlpark, M., Polsa, C., Charoenchai, C., Paengpassa, Y., Kashiwazaki, Y., 1999. Influence of Trypanosoma evansi infection on milk yield of dairy cattle in northeast Thailand. Prev. Vet. Med. 42, 39-44.

Reid, S.A., Husein, A., Hutchinson, G.W., Copeman, D.B., 1999. A possible role for Russa deer (Cervus timorensis russa) and wild pigs in spread of Trypanosoma evansi from Indonesia to Papua New Guinea. Memorias do Instituto Oswaldo Cruz 94, 195-197.

Rurangirwa, F., Musoke, A., Nantulya, V., Tabel, H., 1983. Immune depression in bovine trypanosomiasis: effects of acute and chronic Trypanosama congolense and chronic Trypanosoma vivax infections on antibody response to Brucella abortus vaccine. Parasite Immunol. 5, 267-276.

Sileghem, M., Flyn, J.N., 1992. Suppression of interleukin-2 secretion and interleukin-2 receptor expression during tsetse-transmitted trypansomiasis in cattle. Eur. J. Immunol. 22, 767-773.

Sileghem, M., Darji, A., Remels, L., Hamers, R., De Baetselier, P., 1991. Different mechanisms account for the suppression of interleukin-2 production and the suppression of interleukin-2 receptor expression in Trypanosoma brucei-infected mice. Eur. J. Immunol. 19, 119-124.

Van Meirvenne, N., 1999. Biological diagnosis of human African trypanosomiasis. In: Dumas, M., Bouteille, B., Buguet, A. (Eds.), Progress in human African trypanosomiasis, sleeping sickness. Springer, France, pp. 235-252.

Verloo, D., Holland, W., My, L.N., Thanh, N.G., Tam, P.T., Goddeeris, B., Vercruysse, J., Büscher, P., 2000. Comparison of serological tests for Trypanosoma evansi natural infections in water buffaloes from north Vietnam. Vet. Parasitol. 92, 87-96.

Whitelaw, D., Scott, J.M., Reid, H.W., Holmes, P.H., Jennings, F.W., Urquhart, G.M., 1979. Immunosuppression in bovine trypanosomiasis studies with louping-ill vaccine. Res. Vet. Sci. 26, 102-107. 